\title{
For 2-D Lattice Spin Systems Weak Mixing Implies Strong Mixing
}

\author{
F. Martinelli ${ }^{1}$, E. Olivieri ${ }^{2}$, R.H. Schonmann ${ }^{3}$ \\ ${ }^{1}$ Dipartimento di Matematica, III Università di Roma, Italy. e-mail: martin@mercurio. \\ dm.unirml.it \\ ${ }^{2}$ Dipartimento di Matematica, Università "Tor Vergata" Roma, Italy. e-mail: olivieri \\ @mat.utovrm.it \\ ${ }^{3}$ Mathematics Department, UCLA. Los Angeles, CA 90024, USA. e-mail: rhs@math.ucla.edu
}

Received: 2 June 1993/in revised form: 23 November 1993

\begin{abstract}
We prove that for finite range discrete spin systems on the two dimensional lattice $\mathbf{Z}^{2}$, the (weak) mixing condition which follows, for instance, from the Dobrushin-Shlosman uniqueness condition for the Gibbs state implies a stronger mixing property of the Gibbs state, similar to the Dobrushin-Shlosman complete analyticity condition, but restricted to all squares in the lattice, or, more generally, to all sets multiple of a large enough square. The key observation leading to the proof is that a change in the boundary conditions cannot propagate either in the bulk, because of the weak mixing condition, or along the boundary because it is one dimensional. As a consequence we obtain for ferromagnetic Ising-type systems proofs that several nice properties hold arbitrarily close to the critical temperature; these properties include the existence of a convergent cluster expansion and uniform boundedness of the logarithmic Sobolev constant and rapid convergence to equilibrium of the associated Glauber dynamics on nice subsets of $\mathbf{Z}^{2}$, including the full lattice.
\end{abstract}

\section{Section 0. Introduction}

Let us consider a discrete, finite range lattice spin model in the one phase region and let us analyze the problem of establishing mixing properties of the corresponding Gibbs measure. As is well known, a very powerful approach to the above question is to study the local specifications of the Gibbs measure and to try to derive the uniqueness and mixing properties (e.g. exponential clustering) of the infinite volume state from suitable conditions on the local specifications which express some sort of weak dependence on the boundary conditions. We have in mind, in particular, the Dobrushin [D] and Dobrushin-Shlosman [DS1, DS2, DS3] conditions and we refer the interested reader to a recent paper by two of the authors, [MO1], for a detailed critical review of these conditions together with 
their implications both for the Gibbs measure and for the associated Glauber-type dynamics.

The above conditions can be roughly divided into two classes that we call weak mixing and strong mixing, respectively, according to the following criterion (see Sect. 1 for more details):

A weak mixing condition implies that if in a finite volume $\Lambda$ we consider the Gibbs state with boundary condition $\tau$, then a local (e.g. in a single site $y \in \Lambda^{c}$ ) modification of the boundary condition $\tau$ has an influence on the corresponding Gibbs measure which decays exponentially fast inside $\Lambda$ with the distance from the boundary $\partial \Lambda$.

A strong mixing condition implies, in the same setting as above, that the influence of the perturbation decays in $\Lambda$ exponentially fast with the distance from the support of the perturbation (e.g. the site $y$ ).

This distinction is very important since, even if we are in the one phase region with a unique infinite volume Gibbs state with exponentially decaying truncated correlation functions, it may happen that, if we consider the same Gibbs state in a finite volume $\Lambda$, a local perturbation of the boundary condition radically modifies the Gibbs measure close to the boundary while leaving it essentially unchanged in the bulk and this "long range order effect" at the boundary persists even when $\Lambda$ becomes arbitrarily large. We will refer to this phenomenon as a "boundary phase transition." It is clear that if a "boundary phase transition" takes place, then our Gibbs measure may satisfy a weak mixing condition but not a strong one.

A "boundary phase transition" is apparently not such an exotic phenomenon since, besides being proved for the so-called Czech models [Sh] (in dimension 3 and higher), it is also expected to take place for the 3D ferromagnetic Ising model at low temperatures and small enough magnetic field (depending on the temperature) [DS4].

It is however very reasonable to conjecture that, for finite range interactions, no "boundary phase transition" can take place in 2D for regular enough regions, e.g. squares, for which the boundary is "one dimensional!" In the present paper we prove this conjecture (see Theorem 1.1). It is important to stress that the restriction of the strong mixing condition to squares and other "nice" regions is not a matter of our inability to extend further the result to arbitrary regions. It is actually known that, even in two dimensions and even for ferromagnetic systems, under the same hypothesis of Theorem 1.1, the strong mixing condition may not extend to arbitrarily shaped regions. Some simple counter-examples are presented in [MO1]. From the point of view of the "physics" involved in the problem, it is not surprising that one has to restrict the shape of the regions so that their boundary be basically "one-dimensional."

The main result discussed above is relevant because:

a) A strong mixing condition on e.g. all squares implies some nice consequences for the Gibbs measure (see Sect. 3) like analyticity, the existence of a convergent cluster expansion (see [O] and [OP]), good behavior under a decimation transformation (see [MO2] and [MO3]), existence of a finite logarithmic Sobolev constant (see [MO2] and [LY]), rapid convergence to equilibrium of the associated Glauber dynamics (see [MO1, MO2]). 
b) There are several cases in which the weak mixing condition can be proven to hold. For instance, this is the case if the Dobrushin-Shlosman uniqueness condition [DS1] is verified. In Sect. 3 of this paper we will show that for finite range Ising-like ferromagnets weak mixing indeed holds in most of the onephase region. In particular we can show that it holds in the absence of an external field for all temperatures which are larger than the critical one. As a consequence, we obtain the results quoted in (a) arbitrarily close to the critical temperature. For these models we believe that weak mixing (and hence strong mixing, if the dimension is 2) actually holds for every value of the temperature and external field, except (of course) on the transition line and the critical point.

The paper is organized as follows: in Sect. 1 we define the models, the various mixing conditions and state the main result (Theorem 1.1); in Sect. 2 we prove Theorem 1.1 and, finally, in Sect. 3 we discuss some consequences of the main theorem.

\section{Section 1. General Definitions, Notation and Main Result}

1. The Model. We will consider lattice spin systems in $d$ dimensions (later to be restricted to $d=2$ ) with configuration space of a single spin given by the finite set $S=\{1, \ldots, N\}, N \in \mathbf{N}$. On the lattice $\mathbf{Z}^{\mathbf{d}}$ we will use the norm

$$
\|x\|=\sup _{i=1, \ldots, d}\left|x_{i}\right|
$$

and measure distances accordingly. The configuration space on a subset $\Lambda \subset \mathbf{Z}^{\mathbf{d}}$, namely $S^{\Lambda}$, will be denoted by $\Omega_{\Lambda}$ and a generic element of $\Omega_{\Lambda}$ by $\sigma_{\Lambda}$. If $\Lambda$ coincides with the whole lattice $\mathbf{Z}^{\mathbf{d}}$, then we simply write $\Omega$ and $\sigma$. By $\sigma_{x} \equiv \sigma(x)$ we denote the value of the spin at the site $x \in \Lambda$ in the configuration $\sigma$. Also, when $\Delta \subset \Lambda$, we denote by $\sigma_{\Delta}$ the restriction of $\sigma_{\Lambda}$ to $\Delta$ (note that this is consistent with the previous use of the notation $\sigma_{\Delta}$ ). The number $|X|$ denotes the cardinality of $X \subset \subset \mathbf{Z}^{\mathbf{d}}$ (we write $X \subset \subset \mathbf{Z}^{\mathbf{d}}$ iff $X$ is a finite subset of $\mathbf{Z}^{\mathbf{d}}$ ).

Next we define the potential or interaction $U$ as:

$$
U=\left\{U_{X}, X \subset \subset \mathbf{Z}^{\mathbf{d}}\right\},
$$

where, for every finite $X$,

$$
U_{X}: \Omega \rightarrow \mathbf{R}
$$

satisfies the condition $U_{X}(\sigma)=U_{X}(\eta)$ in case $\sigma_{x}=\eta_{x}$ for all $x \in X$. On the potential $U$ we will always assume the following hypotheses:

H1. Finite range: $\exists r<\infty: U_{X} \equiv 0$ if $\operatorname{diam}(X)>r$.

H2. Translation invariance: $\forall X \subset \subset \mathbf{Z}^{\mathbf{d}} \forall k \in \mathbf{Z}^{\mathbf{d}}$ :

$$
U_{X+k}(\sigma)=U_{X}(\eta) \text { if } \sigma(y+k)=\eta(y) \text { for all } y \in X .
$$

Given $\Lambda \subset \mathbf{Z}^{\mathrm{d}}$ and $\tau \in \Omega_{\Lambda^{\mathrm{c}}}$ (where $\Lambda^{\mathrm{c}}=\mathbf{Z}^{\mathbf{d}} \backslash \Lambda$ ), for every $\sigma \in \Omega$ we denote by $(\sigma \tau)_{\Lambda}$ the configuration obtained from $\sigma$ by changing it to $\tau$ outside $\Lambda$ :

$$
\begin{aligned}
& (\sigma \tau)_{\Lambda}(x)=\sigma(x) \quad \forall x \in \Lambda, \\
& (\sigma \tau)_{\Lambda}(x)=\tau(x) \quad \forall x \in \Lambda^{c} .
\end{aligned}
$$


Given a set $\Lambda \subset \subset \mathbf{Z}^{\mathbf{d}}$, a boundary condition, (b.c.), is a configuration $\tau \in \Omega_{\Lambda^{c}}$ and the energy associated to a configuration $\sigma \in \Omega_{\Lambda}$ when the boundary condition outside $\Lambda$ is $\tau \in \Omega_{\Lambda^{c}}$ is given by:

$$
H_{\Lambda}^{\tau}(\sigma)=H_{\Lambda}(\sigma \mid \tau)=\sum_{X: X \cap A \neq \emptyset} U_{X}\left((\sigma \tau)_{\Lambda}\right) .
$$

Because of the hypothesis $\mathrm{H} 1, H_{\Lambda}^{\tau}(\sigma)$ depends only on $\tau_{x}$ for $x$ in

$$
\partial_{r}^{+} \Lambda=\{x \notin \Lambda: \operatorname{dist}(x, \Lambda) \leqq r\} .
$$

Finally the Gibbs measure in $\Lambda$ with b.c. $\tau \in \Omega_{\Lambda^{c}}$ at inverse temperature $\beta>0$ is the probability measure $\mu_{\Lambda}^{\tau}$ on $\Omega_{\Lambda}$ given by

$$
\mu_{\Lambda}^{\tau}(\sigma)=\frac{\exp \left(-\beta H_{\Lambda}^{\tau}(\sigma)\right)}{Z_{\Lambda}^{\tau}},
$$

where the normalization factor, called partition function, is given by

$$
Z_{\Lambda}^{\tau}=\sum_{\sigma \in \Omega_{\Lambda}} \exp \left(-\beta H_{\Lambda}^{\tau}(\sigma)\right) .
$$

If there exists a unique limiting Gibbs measure for $\Lambda \rightarrow \mathbf{Z}^{\mathbf{d}}$, independent of $\tau$, it will be denoted by $\mu$.

Remark. In the next sections it will be very convenient to consider the Gibbs measure $\mu_{\Lambda}^{\tau}(\sigma)$ directly as a measure on the infinite volume configuration space $\Omega$ such that on $\Lambda^{c}$ we have a.s. $\sigma=\tau$. Moreover we will frequently write, for $\tau$ and $\sigma$ in $\Omega, \mu_{\Lambda}^{\tau}(\sigma)$ instead of the more precise but cumbersome $\mu_{\Lambda}^{\tau_{\Lambda}}\left(\sigma_{\Lambda}\right)$.

2. Definition of Weak and Strong Mixing for the Gibbs Measure. We first recall that the variation distance between two probability measures $\mu_{1}, \mu_{2}$ on a finite set $Y$ is defined as:

$$
\operatorname{Var}\left(\mu_{1}, \mu_{2}\right)=\frac{1}{2} \sum_{y \in Y}\left|\mu_{1}(y)-\mu_{2}(y)\right|=\sup _{X \subset Y}\left|\mu_{1}(X)-\mu_{2}(X)\right| .
$$

More generally, given a metric $\varrho(\cdot, \cdot)$ on a finite space $Y$ (a much more general framework can also be considered) the Kantorovich-Rubinstein-Ornstein-Vasserstein distance with respect to $\varrho$ between two probability measures $\mu_{1}, \mu_{2}$ on $Y$, is defined as

$$
\operatorname{KROV}_{\varrho}\left(\mu_{1}, \mu_{2}\right)=\inf _{\mu \in K\left(\mu_{1}, \mu_{2}\right)} \sum_{y, y^{\prime} \in Y} \varrho\left(y, y^{\prime}\right) \mu\left(y, y^{\prime}\right),
$$

where $K\left(\mu_{1}, \mu_{2}\right)$ is the set of joint representations of $\mu_{1}, \mu_{2}$, namely the set of measures on the cartesian product $Y \times Y$ whose marginals are, respectively, given by $\mu_{1}, \mu_{2}$. i.e., $\mu \in K\left(\mu_{1}, \mu_{2}\right)$ if $\forall B \subset Y$

$$
\begin{aligned}
& \mu(B \times Y)=\sum_{y \in B, y^{\prime} \in Y} \mu\left(y, y^{\prime}\right)=\mu_{1}(B), \\
& \mu(Y \times B)=\sum_{y \in Y, y^{\prime} \in B} \mu\left(y, y^{\prime}\right)=\mu_{2}(B) .
\end{aligned}
$$

It is well known that for the particular case

$$
\varrho\left(y, y^{\prime}\right)=1 \quad \text { iff } y \neq y^{\prime} \quad \text { and } 0 \text { otherwise , }
$$

$\operatorname{KROV}_{\varrho}(\cdot, \cdot)$ coincides with the variation distance $\operatorname{Var}(\cdot, \cdot)$. 
Given a measure $\mu_{\Lambda}$ on $\Omega_{\Lambda}$ we call relativization of $\mu_{\Lambda}$ to $\Omega_{\Delta}$, with $\Delta \subset \Lambda$, the measure $\mu_{\Lambda, \Delta}$ on $\Omega_{\Delta}$ given by

$$
\mu_{\Lambda, \Delta}\left(\sigma_{\Delta}\right)=\sum_{\sigma_{\Lambda \backslash \Delta}} \mu_{\Lambda}\left(\left(\sigma_{\Delta} \sigma_{\Lambda \backslash \Delta}\right)_{\Delta}\right) .
$$

We are now in a position to define strong mixing and weak mixing.

We say that the Gibbs measures $\mu_{\Lambda}$ on $\Omega_{\Lambda}$ satisfy the strong mixing condition with constants $C, \gamma$ if for every subset $\Delta \subset \Lambda$ and every site $y \in \Lambda^{c}$

$$
\sup _{\tau, \tau^{\prime} \in \Omega_{\Lambda^{\prime}}: \tau={ }_{y} \tau^{\prime}} \operatorname{Var}\left(\mu_{\Lambda, \Delta}^{\tau}, \mu_{\Lambda, \Delta}^{\tau^{\prime}}\right) \leqq C e^{-\gamma \operatorname{dist}(\Delta, y)},
$$

where $\tau={ }_{y} \tau^{\prime}$ means that $\tau_{x}=\tau_{x}^{\prime}$ for all $x \neq y$. We denote this condition by $\operatorname{SM}(\Lambda, C, \gamma)$.

We say that the Gibbs measures $\mu_{\dot{A}}$ satisfy the weak mixing condition with constants $C, \gamma$ if, for every subset $\Delta \subset \Lambda$,

$$
\sup _{\tau, \tau^{\prime} \in \Omega_{\Lambda^{c}}} \operatorname{Var}\left(\mu_{\Lambda, \Delta}^{\tau}, \mu_{\Lambda, \Delta}^{\tau^{\prime}}\right) \leqq C \sum_{x \in \Delta, y \in \hat{o}_{r}^{+} \Lambda} \exp (-\gamma\|x-y\|) .
$$

We denote this condition by $W M(\Lambda, C, \gamma)$.

Finally we say that $W M_{\varrho}(\Lambda, C, \gamma)$ holds if $\forall \Delta \subset \Lambda$,

$$
\sup _{\tau, \tau^{\prime} \in \Omega_{\Lambda^{c}}} \operatorname{KROV}_{\varrho}\left(\mu_{\Lambda, \Delta}^{\tau}, \mu_{\Lambda, \Delta}^{\tau^{\prime}}\right) \leqq C \sum_{x \in \Delta, y \in \partial_{r}^{+} \Lambda} \exp (-\gamma\|x-y\|) \text {. }
$$

3. The Main Result. The cube (we will also call it "square" if $d=2$ ) of side $2 L+1$ centered at the origin is the set

$$
\Lambda_{L}=\left\{x \in \mathbf{Z}^{\mathbf{d}} ;\|x\| \leqq L\right\}
$$

Our main result reads as follows:

Theorem 1.1. In 2 dimensions, if there exist positive constants $C$ and $\gamma$ such that the Gibbs measures $\mu_{\dot{\Lambda}}$ satisfy the weak mixing condition $\operatorname{WM}(\Lambda, C, \gamma)$ for all $\Lambda \subset \subset \mathbf{Z}^{2}$, then there exist positive constants $C^{\prime}$ and $\gamma^{\prime}$ such that they also satisfy the strong mixing condition $\operatorname{SM}\left(\Lambda_{L}, C^{\prime}, \gamma^{\prime}\right)$ for every square $\Lambda_{L}$.

Remark. Actually, as we will see in the next section, the conclusion of the above theorem remains true even if we assume the weak mixing not for all finite subsets of $\mathbf{Z}^{\mathbf{d}}$ but only for all subsets of a square $\Lambda_{L_{0}}$ provided that $L_{0}$ is large enough (depending of course on the constants $C$ and $\gamma$ and on the range of the interaction). Moreover it is possible to show (see [MO1]) that, once $\operatorname{SM}\left(\Lambda_{L}, C^{\prime}, \gamma^{\prime}\right)$ holds for every square $\Lambda_{L}$, then it also holds for all sets "multiple" (i.e., union of disjoint translates) of a given, large enough, square.

\section{Section 2. Proof of Theorem 1.1}

Theorem 1.1 actually follows from an apparently weaker result that we state as a proposition. First we need to introduce a technical definition; given $L$ and $\tau$, $\tau^{\prime} \in \Omega_{\Lambda_{L}}^{c}$ set

$$
Q_{L, \tau, \tau^{\prime}}=\left\{x \in \Lambda_{L} ;\|x-y\| \geqq L^{1 / 2} \quad \forall y \text { such that } \tau(y) \neq \tau^{\prime}(y)\right\} .
$$


Proposition 2.1. In 2 dimensions, if there exist positive constants $C$ and $\gamma$ such that the Gibbs measures $\mu_{\dot{\Lambda}}$ satisfy the weak mixing condition $\operatorname{WM}(\Lambda, C, \gamma)$ for every $\Lambda \subset \subset \mathbf{Z}^{2}$, then there exist positive constants $C_{0}$ and $\gamma_{0}$ such that for every square $\Lambda_{L}$ and every site $y \in \Lambda_{L}^{c}$,

$$
\sup _{\tau, \tau^{\prime} \in \Omega_{\Lambda_{L}^{\prime}}: \tau={ }_{y} \tau^{\prime}} \operatorname{Var}\left(\mu_{\Lambda_{L}, Q_{L}, \tau, \tau^{\prime}}^{\tau}, \mu_{\Lambda_{L}, Q_{L}, \tau, \tau^{\prime}}^{\tau^{\prime}} \leqq C_{0} e^{-\gamma_{0} L^{1 / 4}}\right.
$$

From the above proposition and from the triangle-inequality property of the variation distance, it follows immediately that, if $\tau$ and $\tau^{\prime}$ are two arbitrary boundary conditions outside the square $\Lambda_{L}$, then we have

$$
\operatorname{Var}\left(\mu_{\Lambda_{L}, Q_{L}, \tau, \tau^{\prime}}^{\tau}, \mu_{\Lambda_{L}, Q_{L}, \tau, \tau^{\prime}}^{\tau^{\prime}} \leqq C_{0} 4 r(L+2 r) e^{-\gamma_{0} L^{1 / 4}},\right.
$$

where $r$ is the range of the interaction. We can use at this point the following result which was proved in Sect. 4 of [MO1]. (See the proof of Theorem 4.1 there, and observe that the only way in which the hypothesis of strong mixing for some cube was used there was through their inequality (4.2). It is well known that the uniform estimate on the spectral gap obtained in that theorem implies the type of strong mixing that we claim; this implication is contained for instance in Proposition 4.1 in [MO1].) We quote the result in our special 2D setting.

Theorem 2.1. In 2 dimensions, there exists a constant $\bar{L}$ such that if

$$
\sup _{\tau, \tau^{\prime} \in \Omega_{\Lambda_{L_{0}}^{c}}} \operatorname{Var}\left(\mu_{\Lambda_{L_{0}}, Q_{L_{0}, \tau, \tau^{\prime}}}, \mu_{\Lambda_{L_{0}}^{\tau^{\prime}}, Q_{L_{0}, \tau, \tau^{\prime}}}^{\tau^{\prime}} \leqq L_{0}^{-d-2}\right.
$$

for some $L_{0}>\bar{L}$, then there exist positive constants $C$ and $\gamma$ such that the strong mixing condition $\operatorname{SM}\left(\Lambda_{L}, C, \gamma\right)$ holds for all squares $\Lambda_{L}, L \geqq 0$.

From (2.1) (where $L$ is arbitrary) and the above theorem we immediately get Theorem 1.1.

Remarks. Actually in order to be able to use the results of [MO1] we only need to prove the result of the proposition for $L$ so large that the r.h.s. of (2.1) is smaller than $\frac{K}{L^{4}}$, where $K$ is a suitable constant. As one can easily check in the proof given below, this requires as input the weak mixing condition only for all subsets of a large enough square.

It should be stressed that the powers of $L$ that appear in (2.1) and (2.2) are technical and have no physical meaning.

Thus we are left with the proof of Proposition 2.1. Suppose that $L, y \in \Lambda_{L}^{c}, \tau$ and $\tau^{\prime}$ are fixed and such that $\tau={ }_{y} \tau^{\prime}$, and abbreviate $Q=Q_{L}, \tau, \tau^{\prime}=\left\{x \in \Lambda_{L} ;\|x-y\| \geqq\right.$ $\left.L^{1 / 2}\right\}$. The key idea is to show that it is possible to construct, by successive "improvements" over an increasing sequence of length scales, a coupling,

$$
v \in K\left(\mu_{\Lambda_{L}, Q}^{\tau}, \mu_{\Lambda_{L}, Q}^{\tau^{\prime}}\right)
$$

of $\mu_{\Lambda_{L}, Q}^{\tau}$ and $\mu_{\Lambda_{L}, Q}^{\tau^{\prime}}$ such that

$$
v(\sigma \neq \eta) \leqq C_{0} e^{-\gamma_{0} L^{1 / 4}}
$$

for suitable positive constants $C_{0}$ and $\gamma_{0}$, which do not depend on $L, y, \tau$ and $\tau^{\prime}$. Clearly (2.3) proves the proposition, because of the remark concerning (1.8).

Each "improvement" will be carried out via the so-called "surgery" technique (used for instance in [DS1]) that we will now recall (here it is very convenient to adopt the convention pointed out in the remark which followed (1.5)). 
The ingredients of a surgery are:

i) A finite set $\Lambda \subset \subset \mathbf{Z}^{\text {d }}$ and a subset $\Gamma \subset \Lambda$.

ii) A coupling $v \in K\left(\mu_{\Lambda}^{\tau_{1}}, \mu_{\Lambda}^{\tau_{2}}\right)$ for some $\tau_{1}$ and $\tau_{2}$.

iii) A transition kernel $T(\eta, \xi ; \bar{\eta}, \bar{\xi}$ ) (where $\eta, \xi, \bar{\eta}, \bar{\xi}$ run over $\Omega$ ) which has to satisfy the following property: For each pair $\eta, \xi$

$$
T(\eta, \xi ;,) \in K\left(\mu_{\Gamma}^{\eta}, \mu_{\Gamma}^{\xi}\right) .
$$

In particular, $T(\eta, \xi ; \bar{\eta}, \bar{\xi})=0$ if $\eta_{\Gamma^{c} \neq} \bar{\eta}_{\Gamma^{c}}$ or $\xi_{\Gamma^{c}} \neq \bar{\xi}_{\Gamma^{c}}$.

The result of a surgery is a new coupling $\bar{v} \in K\left(\mu_{\Lambda}^{\tau_{1}}, \mu_{\Lambda}^{\tau_{2}}\right)$ defined by:

$$
\bar{v}(\bar{\eta}, \bar{\xi}) \equiv \sum_{\eta, \xi} v(\eta, \xi) T(\eta, \xi ; \bar{\eta}, \bar{\xi}) \text {. }
$$

One can check that the measure $\bar{v}$ defined above is indeed a new coupling of $\mu_{\Lambda}^{\tau_{1}}$ and $\mu_{\Lambda}^{\tau_{2}}$ with the following simple type of computation:

$$
\begin{aligned}
\sum_{\bar{\xi}} \bar{v}(\bar{\eta}, \bar{\xi}) & =\sum_{\eta, \xi} v(\eta, \xi) \sum_{\bar{\xi}} T(\eta, \xi ; \bar{\eta}, \bar{\xi})=\sum_{\eta, \xi} v(\eta, \xi) \mu_{\Gamma}^{\eta}(\bar{\eta}) \\
& =\sum_{\eta} \mu_{\Lambda}^{\tau_{1}}(\eta) \mu_{\Gamma}^{\eta}(\bar{\eta})=\mu_{\Lambda}^{\tau_{1}}(\bar{\eta})
\end{aligned}
$$

where the second equality follows from the property (iii) above, while the third and fourth equalities follow from the definition of coupling and the DLR equations, respectively.

The simplest example is the so-called product surgery defined by:

$$
T(\eta, \xi ; \bar{\eta}, \bar{\xi})=\mu_{\Gamma}^{\eta}(\bar{\eta}) \mu_{\Gamma}^{\xi}(\bar{\xi}) .
$$

Let us now go back to the proof of Proposition 2.1. Recall that $y \in \Lambda_{L}^{c}$ is fixed. Given an integer $l_{0}$ to be chosen conveniently later on independently of $L$ (in (a) below), we define for each integer $i=1,2, \ldots,\left[\frac{L^{1 / 2}}{L^{1 / 4}}\right]$,

$$
\begin{aligned}
& \Gamma_{i} \equiv\left\{x \in \Lambda_{L} ;\|x-y\|>(i-1) L^{1 / 4}\right\}, \\
& D_{i} \equiv\left\{x \in \Lambda_{\bar{L}} ; i L^{1 / 4}-r<\|x-y\| \leqq i L^{1 / 4}\right\}, \\
& A_{i} \equiv\left\{x \in D_{i} ; \min _{z \notin \Lambda_{L}}\|x-z\| \leqq l_{0}\right\}, \\
& B_{i} \equiv D_{i} \backslash A_{i} .
\end{aligned}
$$

It is easily seen that for large $L$ there can be at most one value of $i$ for which the set $D_{i}$ is at a distance less than $L^{1 / 4} / 2$ from one of the four vertices of the square $\Lambda_{L}$. If such a value of $i$ exists, we will denote it by $i_{0}$, and below we will have to treat it differently than the other values of $i$.

The following two geometric facts can be easily checked:

a) Let $C$ and $\gamma$ be the positive constants which appear in the statement of the proposition. We can choose $l_{0}$ so large (depending only on $C, \gamma$ and $r$ ) that for all large $L$ and each $i \neq i_{0}$,

$$
C \sum_{u \in B_{i}, v \in \partial_{r}^{+} \Gamma_{i}} \exp (-\gamma\|u-v\|) \leqq 1 / 2 .
$$


b) If $L$ is large enough, then for each $i \neq i_{0}$,

$$
\left|A_{i}\right|=2 l_{0} r .
$$

The key idea in the proof is to iterate the procedure described below for $i=1,2, \ldots,\left[\frac{L^{1 / 2}}{L^{1 / 4}}\right]$. We describe now the $i$-th step of the iteration. Suppose that from steps $1, \ldots, i-1$ we have been able to construct a joint representation of $\mu_{\Lambda_{L}}^{\tau}$, $\mu_{\Lambda_{L}}^{\tau^{\prime}}$ that we denote by $v_{i-1}$. At the $i$-th step we obtain a new coupling $v_{i} \in$ $K\left(\mu_{\Lambda_{L}}^{\tau_{1}}, \mu_{\Lambda_{L}}^{\tau_{2}}\right)$ as follows:

I) If $i=i_{0}$ we do nothing, namely we set

$$
v_{i_{0}}=v_{i_{0}-1} .
$$

II) If, on the contrary, $i \neq i_{0}$, then we modify $v_{i-1}$ via the following surgery applied to $v_{i-1}$ on the set $\Gamma_{i}$. The transition kernel of the surgery, $T_{i}$, is defined as follows: define

II.i) If $\eta_{D_{i-1}}=\xi_{D_{t-1}}$, then keep in mind that the range of interaction is $r$ and

$$
\begin{aligned}
& T_{i}(\eta, \xi ; \bar{\eta}, \bar{\xi})=\mu_{\Gamma_{i}}^{\eta}(\bar{\eta}) \quad \text { if } \bar{\eta}_{\Gamma_{i}}=\bar{\xi}_{\Gamma_{i}} \text { and } \bar{\xi}_{\Gamma_{i}^{c}}=\xi_{\Gamma_{i}^{c}}, \bar{\eta}_{\Gamma_{i}^{c}}=\eta_{\Gamma_{i}^{c}}, \\
& T_{i}(\eta, \xi ; \bar{\eta}, \bar{\xi})=0 \text { otherwise }
\end{aligned}
$$

II.ii) If $\eta_{D_{i-1}} \neq \xi_{D_{i-1}}$, then we observe that, since $i \neq i_{0}$, we can use (a) above and the weak mixing property on the set $\Gamma_{i}$ to deduce that there exists $\tilde{v}_{i} \in K\left(\mu_{\Gamma_{i}}^{\eta}, \mu_{\Gamma_{i}}^{\xi}\right)$ such that:

$$
\tilde{v}_{i}\left((\tilde{\eta}, \tilde{\xi}) ; \tilde{\eta}_{B_{i}}=\tilde{\xi}_{B_{i}}\right) \geqq 1 / 2,
$$

for all $L$ large enough (independently of $i$ ) provided that $l_{0}$ was chosen properly. In this case we define $T_{i}(\eta, \xi ; \bar{\eta}, \bar{\xi})$ as:

$$
T_{i}(\eta, \xi ; \bar{\eta}, \bar{\xi})=\sum_{\tilde{\eta}, \tilde{\xi}} \tilde{v}_{i}(\tilde{\eta}, \tilde{\xi}) \mu_{A_{i}}^{\tilde{\eta}}(\bar{\eta}) \mu_{A_{t}}^{\tilde{\xi}}(\bar{\xi}) .
$$

(In (2.9) we are taking the composition of two surgeries, the second of which is the product surgery on $A_{i}$.)

It is easy to check that indeed $T_{i}(\eta, \xi ; \bar{\eta}, \bar{\xi})$, defined by (II.i) and (II.ii), has the right properties to be the kernel of a surgery; moreover, thanks to (2.7), (2.8), (2.9) and (b) above, the following crucial estimate holds:

$$
\inf _{\eta, \bar{\xi}, \bar{\eta} ; \bar{\eta}_{D_{i}}=\bar{\xi}_{D_{i}}} T_{i}(\eta, \xi ; \bar{\eta}, \bar{\xi}) \geqq \frac{1}{2} a^{2\left|A_{\imath}\right|}=\frac{a^{4 r l_{0}}}{2} \equiv \delta>0,
$$

where the constant $a>0$ depends only on the range and the norm of the interaction $U$.

Remark. Notice that the constant $\delta$ is independent of the side $L$ and of $i$ just because we are working in two dimensions; it is only in two dimensions in fact that the cardinality of the sets $A_{i}$, can be bounded above uniformly in $L$ and $i$ (by $2 r l_{0}$ )!

Finally we set, in case (II), according to (2.4),

$$
v_{i}(\bar{\eta}, \bar{\xi}) \equiv \sum_{\eta, \xi} v_{i-1}(\eta, \xi) T_{i}(\eta, \xi ; \bar{\eta}, \bar{\xi}) .
$$


Let now $i_{Q}$ be the largest $i$ such that $Q \subset \Gamma_{i}$. Then $v_{i_{Q}} \in K\left(\mu_{\Lambda_{L}}^{\tau}, \mu_{\Lambda_{L}}^{\tau^{\prime}}\right.$, ) and

$$
v_{i_{Q}}\left((\eta, \xi) ; \eta_{Q} \neq \xi_{Q}\right) \leqq \prod_{i=2, \ldots, i_{Q}, i \neq i_{0}} v_{i}\left((\eta, \xi) ; \eta_{D_{i}} \neq \xi_{D_{i}}\right) \leqq(1-\delta)^{i_{Q}-3} .
$$

Clearly (2.12) implies (2.3) and hence proves Proposition 2.1 and therefore also Theorem 1.1 .

Remark. We notice that in the above proof we used the weak mixing condition only for the sets $\Gamma_{i}$ which, in turn, can have only two particular geometric shapes. Actually we can weaken our hypotheses even further if we use the results of Appendix 2 in [MO1]. There it was shown that, in order to prove strong mixing, it is sufficient to establish, in a large enough square (or hypercube in $d$-dimensions), a bound like (2.1) on the truncated correlation functions of two local observables $f$ and $g$ with support of diameter equal to the range $r$ of the interaction and located on two opposite faces of the square. Using this geometry it is easy to show that one could modify slightly the geometric construction used in the proof, in order to require the weak mixing condition only on rectangles.

\section{Section 3. Some Consequences and Applications}

In this final section we first prove the equivalence, in $2 \mathrm{D}$, of the validity of weak mixing with several other statements that involve either the Gibbs states or a Glauber dynamics reversible with respect to them; afterwards we check that weak mixing actually holds true in most part of the one phase region of Ising-like ferromagnets. In order to state our result we first have to give some definitions.

A Glauber dynamics is defined by means of its generator $L$ which is formally given by:

$$
L f(\sigma)=\sum_{x, a} c_{x}(\sigma, a)\left(f\left(\sigma^{x, a}\right)-f(\sigma)\right),
$$

where $\sigma^{x, a}$ is the configuration obtained from $\sigma$ by setting the spin at $x$ equal to the value $a \in S$ and the non-negative quantities $c_{x}(\sigma, a)$ are called "jump rates."

We will also consider the dynamics associated to the above described jump rates in a finite volume $\Lambda$ with boundary conditions $\tau$ outside $\Lambda$. By this we mean the dynamics on $\Omega_{\Lambda}$ generated by $L_{\Lambda}^{\tau}$, defined by

$$
L_{\Lambda}^{\tau} f(\sigma)=\sum_{x \in \Lambda, a} c_{x}^{\tau, \Lambda}(\sigma, a)\left(f\left(\sigma^{x, a}\right)-f(\sigma)\right)
$$

with

$$
c_{x}^{\tau, \Lambda}(\sigma, a) \equiv c_{x}\left((\sigma \tau)_{\Lambda}, a\right) .
$$

The general hypotheses on the jump rates, that we shall always assume, are the following ones.

H1. Finite range $r$. This means that there exists a finite $r$ such that for each $x \in Z^{\mathbf{d}}$, if the configurations $\sigma$ and $\eta$ satisfy $\eta(y)=\sigma(y)$ whenever $\|y-x\| \leqq r$, then for every $a \in S$,

$$
c_{x}(\sigma, a)=c_{x}(\eta, a),
$$


H2. Translation invariance. That is, if for some $x \in \mathbf{Z}^{\mathbf{d}}$ we have for every $y \in Z^{\mathbf{d}}$ that $\eta(y)=\sigma(y+x)$, then we also have for every $y \in \mathbf{Z}^{\mathbf{d}}$ and every $a \in S$,

$$
c_{y+x}(\sigma, a)=c_{x}(\eta, a) .
$$

H3. Positivity and boundedness. There exist two positive constants $k_{1}, K_{2}$ such that

$$
0<k_{1} \leqq \inf _{\sigma, x, a} c_{x}(\sigma, a) \leqq \sup _{\sigma, x, a} c_{x}(\sigma, a) \leqq k_{2} .
$$

H4. Reversibility with respect to the Gibbs measures. For each $x \in \mathbf{Z}^{\mathbf{d}}$ and $a \in S$,

$$
\exp \left(-\beta \sum_{X x} U_{X}(\sigma)\right) c_{x}(\sigma, a)=\exp \left(-\beta \sum_{X x_{x}} U_{X}\left(\sigma^{x, a}\right)\right) c_{x}\left(\sigma^{x, a}, \sigma_{x}\right)
$$

Under these conditions above $L$ (resp. $L_{A}^{\tau}$ ) generates a unique positive selfadjoint contraction semigroup on the space $L^{2}(\Omega, d \mu)\left(\operatorname{resp} . L^{2}\left(\Omega_{\Lambda}, d \mu_{\Lambda}^{\tau}\right)\right)$ that will be denoted by $P_{t}\left(\right.$ resp. $\left.P_{t}^{\Lambda, \tau}\right)$ (see [L]).

It is immediate to check that, in finite volume, reversibility implies that the unique invariant measure of the dynamics coincides with the Gibbs measure $\mu_{\Lambda}^{\tau}$.

Given a family (finite or infinite) $\Gamma$ of subsets of the lattice $\mathbf{Z}^{\mathbf{d}}$ we say that $E C U(\Gamma, m)$ holds if for each cylindrical (i.e., depending on finitely many spins) $f: \Omega \rightarrow R$ there exists a finite constant $C_{f}$ such that:

$$
\sup _{\tau, \Lambda \in \Gamma}\left\|P_{t}^{\tau, \Lambda} f-\mu_{\Lambda}^{\tau}(f)\right\|_{\infty} \leqq C_{f} \exp (-m t) .
$$

Below we will use $v(f)$ to denote the average of the continuous function $f: \Omega \rightarrow \mathbf{R}$ with respect to the measure $v$. (This is a common abuse of notation, since $v(\sigma)$ still denotes the $v$-probability assigned to the configuration $\sigma$.) Using this notation we define $\operatorname{gap}(\Lambda, \tau)$ as:

$$
\operatorname{gap}(\Lambda, \tau) \equiv \inf _{f: \mu_{\Lambda}^{\tau}(f)=0, \mu_{\Lambda}^{\tau}\left(f^{2}\right)=1} \frac{1}{2} \sum_{x, a, \sigma \in \Omega_{\Lambda}} \mu_{\Lambda}^{\tau}(\sigma) c_{x}(\sigma, a)\left(f\left(\sigma^{x, a}\right)-f(\sigma)\right)^{2} .
$$

We define the Logarithmic Sobolev Constant $c_{s}(v)$ for an arbitrary measure $v$ on $\Omega_{\Lambda}$ as the smallest number $c$ such that for every non-negative continuous function $f: \Omega_{\Lambda} \rightarrow \mathbf{R}$ the following inequality holds:

$$
v\left(f^{2} \log (f)\right) \leqq c v\left(\sum_{x \in \Lambda}\left(\partial_{\sigma_{x}} f(\sigma)\right)^{2}\right)+v\left(f^{2}\right) \log \left(\left(v\left(f^{2}\right)\right)^{1 / 2}\right),
$$

where

$$
\partial_{\sigma_{x}} f(\sigma)=\frac{f\left(\sigma^{x, i+1}\right)-f(\sigma)}{2}
$$

if $\sigma_{x}=i$, with $N+1 \equiv 1$.

We define the decimation transformation of spacing $b, T_{b}$, as follows. Let

$$
\mathbf{Z}^{\mathbf{d}}(b) \equiv\left\{y \in \mathbf{Z}^{\mathbf{d}} ; y=b x \text { for some } x \in \mathbf{Z}^{\mathbf{d}}\right\} \text {. }
$$

Then

$$
T_{b} \mu_{\Lambda}^{\tau} \equiv \mu_{\Lambda, \mathbf{Z}^{\mathrm{d}}(b) \cap \Lambda}^{\tau},
$$

i.e., $T_{b} \mu_{\Lambda}^{\tau}$ is the relativization to $\mathbf{Z}^{\mathbf{d}}(b) \cap \Lambda$ of $\mu_{\Lambda}^{\tau}$. 
Now we recall the Dobrushin-Shlosman finite size condition for uniqueness. Given a metric $\varrho$ on the single spin space $S$ let

$$
\varrho_{\Lambda_{0}}(\sigma, \eta)=\sum_{x \in \Lambda_{0}} \varrho(\sigma(x), \eta(x)) \text {. }
$$

Then we say that condition $D S U_{\varrho}\left(\Lambda_{0}, \delta\right)$ is satisfied if there exists a finite set $\Lambda_{0} \subset \subset \mathbf{Z}^{\mathbf{d}}$, and a number $\delta>0$ such that to every $y \in \Lambda_{0}^{c}$ we can associate a number $\alpha_{y}$ which satisfies the following two inequalities: $\forall \tau, \tau^{\prime} \in \Omega_{\Lambda_{0}}^{c}$ with $\tau_{x}^{\prime}=\tau_{x} \forall x \neq y$

$$
\operatorname{KROV}_{\varrho_{\Lambda_{0}}}\left(\mu_{\Lambda_{0}}^{\tau}, \mu_{\Lambda_{0}}^{\tau^{\prime}}\right) \leqq \alpha_{y \varrho}\left(\tau_{y}, \tau_{y}^{\prime}\right)
$$

and

$$
\sum_{y \in \partial_{r}^{+} \Lambda_{0}} \alpha_{y} \leqq \delta\left|\Lambda_{0}\right|
$$

We simply say that $\operatorname{DSU}\left(\Lambda_{0}, \delta\right)$ is satisfied if (3.3) and (3.4) hold with $\varrho(i, j)$ given by (1.8).

Theorem 3.1 (Dobrushin-Shlosman [DS1]). If $\operatorname{DSU}_{\varrho}\left(\Lambda_{0}, \delta\right)$ is satisfied for some $\varrho$, $\Lambda_{0}$ and $\delta<1$, then there exist positive constants $C$ and $\gamma$ such that condition $\mathrm{WM}_{\varrho}(\Lambda, C, \gamma)$ (defined by (1.12)) holds for every $\Lambda \subset \subset \mathbf{Z}^{\mathbf{d}}$. In particular, if $\varrho(i, j)$ is defined by (1.8), we obtain $\mathrm{WM}(\Lambda, C, \gamma)$ for all $\Lambda \subset \subset \mathbf{Z}^{\mathrm{d}}$.

We are now in a position to state the main consequence of Theorem 1.1:

Theorem 3.2. In 2 dimensions the following are equivalent:

(i) There exist positive constants $C$ and $\gamma$ such that $\operatorname{WM}(\Lambda, C, \gamma)$ holds for every $\Lambda \subset \subset \mathbf{Z}^{2}$.

(ii) There exist positive constants $C$ and $\gamma$ such that $\operatorname{SM}\left(\Lambda_{L}, C, \gamma\right)$ holds for every square $\Lambda_{L}, L>0$.

(iii) There exists a positive constant $m$ such that $\operatorname{ECU}(\Gamma, m)$ holds, with $\Gamma$ the family of all squares in $\mathbf{Z}^{\mathbf{2}}$.

(iv) There exists a positive constant $m$ such that $\operatorname{gap}\left(\Lambda_{L}, \tau\right) \geqq m$ for every square $\Lambda_{L}$, $L>0$.

(v) There exists a positive constant $c_{0}$ such that $\sup _{\tau} c_{s}\left(\mu_{\Lambda_{L}}^{\tau}\right) \leqq c_{0}$ for every square $\Lambda_{L}$, $L>0$.

(vi) $\operatorname{DSU}\left(\Lambda_{0}, \delta\right)$ is satisfied for some $\Lambda_{0} \subset \subset \mathbf{Z}^{2}$ and $\delta<1$.

Moreover each one of the above conditions implies that the renormalized measure $T_{b} \mu$ converges (exponentially fast in $b$ ) to a product measure and that it is possible to carry out a convergent cluster expansion for $\mu_{\Lambda}^{\tau}$.

Remarks. For simplicity we did not state the above result in its strongest version; namely in (i) we could have assumed weak mixing only for all subsets of a large enough square or, even, only for all rectangles with large enough shortest side inside a large enough square. Similarly, in the remaining conditions, the collection of all squares could have been replaced by the collection of all subsets of $\mathbf{Z}^{\mathbf{d}}$ multiple of a large enough square.

It is also worthwhile to stress the fact that from items (iii), (iv) and (v) of the theorem, analogous statements follow for the system on the infinite lattice $\mathbf{Z}^{\mathbf{d}}$.

There is actually only one step in the proof of Theorem 3.2 where we use the hypothesis that $d=2$ : to assure that Theorem 1.1 can be used and hence (i) implies (ii). In arbitrary dimension it is still true (changing, of course $\mathbf{Z}^{\mathbf{2}}$ to $\mathbf{Z}^{\mathbf{d}}$ and squares 
to cubes in the statements) that (ii), (iii), (iv) and (v) above are equivalent, that they imply (vi) and that (vi) implies (i).

Proof. That (ii), and (iv) are equivalent was proved in [MO1]. Moreover (iii) clearly implies (iv) and (ii) implies (v) (see [MO2]) which, because of hypercontractivity (see e.g. [G] or [SZ]), implies (iii). That (i) implies (ii) was proved in Theorem 1.1, while the Dobrushin-Shlosman uniqueness theorem above, Theorem 3.1, tells us that (vi) implies (i). Therefore we only have to show that (ii) implies (vi). The simple argument that we present below to prove this is borrowed from the proof of Theorem 3.3 in [DS1]; we reproduce it here, for the reader's convenience, because that theorem was stated in a weaker form in [DS1]. The argument is presented in arbitrary dimension. Let $\Lambda_{0}$ be a cube of side $L_{0}$ and, for $y \in \partial_{r}^{+} \Lambda_{0}$ and $A>0$ set

$$
B_{A}(y) \equiv\left\{x \in \Lambda_{0} ;\|x-y\| \geqq A \log \left(L_{0}\right)\right\} .
$$

Suppose that $\tau^{\prime}={ }_{y} \tau$. Because of the validity of $\operatorname{SM}\left(\Lambda_{0}, C, \gamma\right)$ there exists a joint representation $v$ of $\mu_{\Lambda_{0}, B_{A}(y)}^{\tau}$ and $\mu_{\Lambda_{0}, B_{A}(y)}^{\tau^{\prime}}$ such that

$$
\sum_{z \in B_{A}(y)} v((\sigma, \eta) ; \sigma(z) \neq \eta(z)) \leqq L_{0}^{d} v((\sigma, \eta) ; \sigma \neq \eta) \leqq L_{0}^{d} C \exp \left(-\gamma A \log \left(L_{0}\right)\right) .
$$

Let now $q\left(\sigma_{\Lambda_{0} \backslash B_{A}(y)}, \sigma_{\Lambda_{0} \backslash B_{A}(y)}^{\prime} \mid \sigma_{B_{A}(y)}, \sigma_{B_{A}(y)}^{\prime}\right)$ be any joint representation of $\mu_{\Lambda_{0} \backslash B_{A}(y)}^{(\sigma \tau) B_{A}(y)}$ and $\mu_{\Lambda_{0} \backslash B_{A}(y)}^{\left(\sigma^{\prime} \tau^{\prime}\right) B_{A}(y)}$. Then, as one can easily verify, the measure

$$
\tilde{v}\left(\sigma, \sigma^{\prime}\right)=q\left(\sigma_{\Lambda_{0} \backslash B_{A}(y)}, \sigma_{\Lambda_{0} \backslash B_{A}(y)}^{\prime} \mid \sigma_{B_{A}(y)}, \sigma_{B_{A}(y)}^{\prime}\right) v\left(\sigma_{B_{A}(y)}, \sigma_{B_{A}(y)}^{\prime}\right)
$$

is a joint representation of $\mu_{\Lambda_{0}}^{\tau}$ and $\mu_{\Lambda_{0}}^{\tau^{\prime}}$ and its relativization to the set $B_{A}(y) \times B_{A}(y)$ coincides with the measure $v$. Thus, because of the definition of $\mathrm{KROV}_{\varrho \Lambda_{0}}$ and using (3.5) and (3.6), we have, when $\varrho$ is given by (1.8),

$$
\mathrm{KROV}_{\varrho \Lambda_{0}}\left(\mu_{\Lambda_{0}}^{\tau}, \mu_{\Lambda_{0}}^{\tau^{\prime}}\right) \leqq L_{0}^{d} C \exp \left(-\gamma A \log \left(L_{0}\right)\right)+3^{d}\left(A \log \left(L_{0}\right)\right)^{d} .
$$

Clearly, if we take $A=d / \gamma$ and if $L_{0}$ is taken large enough, the r.h.s. of (3.7) is bounded above by $\left(3^{d}+1\right)\left(A \log \left(L_{0}\right)\right)^{d} \equiv \alpha_{y}$. Thus, in this case,

$$
\sum_{y \in \partial_{r}^{+} \Lambda_{0}} \alpha_{y} \leqq\left(3^{d}+1\right)\left(A \log \left(L_{0}\right)\right)^{d}\left|\partial_{r}^{+} \Lambda_{0}\right| \ll\left|\Lambda_{0}\right|
$$

if $L_{0}$ is large enough. This finishes the proof that (ii) implies (vi).

The fact that condition (ii) implies that the renormalized measure $T_{b} \mu$ converges (exponentially fast in $b$ ) to a product measure and that is possible to carry out a convergent cluster expansion for $\mu_{\Lambda}^{\tau}$ follows directly from the methods developed in $[\mathrm{O}, \mathrm{OP}, \mathrm{MO} 1]$ (this was already noticed in [MO3] in the particular case of 3D Ising model but the argument is valid for the class of interactions considered in this paper).

In fact in $[\mathrm{O}, \mathrm{OP}]$ a polymer expansion was performed via a block decimation procedure on a sufficiently large scale. The fact that the convergence of the corresponding cluster expansion immediately follows from the validity of $\operatorname{SM}(\Lambda, C, \gamma)$ on a suitable cube $\Lambda$ was shown in Appendix 2 of [MO1].

From this point on, we restrict ourselves to the case $S=\{-1,+1\}$ and consider the (formal) Ising-type Hamiltonian:

$$
H(\sigma)=-\frac{1}{2} \sum_{x \neq y} J(x-y) \sigma_{x} \sigma_{y}-\sum_{x} h \sigma_{x},
$$


where $J(\cdot)$ satisfies the following conditions for arbitrary $z \in \mathbf{Z}^{\mathbf{d}}: J(-z)=J(z)$, $J(z) \geqq 0$ and in case $\|z\|>r$, then $J(z)=0$. More formally, the corresponding potential $U=\left\{U_{X}, X \subset \subset \mathbf{Z}^{\mathbf{d}}\right\}$ is given by $U_{X}(\sigma)=-h \sigma_{x}$ for $X=\{x\}, \quad U_{X}(\sigma)=$ $-J(x-y) \sigma_{x} \sigma_{y}$ for $X=\{x, y\}$, and $U_{X}=0$ otherwise. For arbitrary $\Lambda \subset \subset Z^{\mathrm{d}}$ we will use the notation $\mu_{\Lambda}^{+}$(resp. $\mu_{\Lambda}^{-}$) to denote the Gibbs measure in $\Lambda$ with b.c. identically +1 (resp. -1 ). By $\beta_{c}$ we will denote the critical inverse temperature, i.e., the supremum of the values of the inverse temperature $\beta$ (in the definition (1.4)) for which there is a unique Gibbs measure on the infinite lattice $\mathbf{Z}^{\mathbf{d}}$, when $h=0$. The following theorem shows that the weak mixing condition is verified in most of the phase space (the $(\beta, h)$ plane) of these models. As a consequence, in $d=2$ the equivalent statements (i) -(vi) in Theorem 3.2 all hold for these same values of $\beta$ and $h$. By "standard Ising model" below we refer, as usual, to the particular case in which $J(z)=1$ iff $z$ is one of the $2 d$ nearest neighbors (in the usual sense, i.e., w.r.t. the Euclidean norm) of the origin in $\mathbf{Z}^{\mathbf{d}}$, and $J(z)=0$ otherwise.

Theorem 3.3. For every Hamiltonian of the form (3.9), in arbitrary dimension:

a) The following two statements are equivalent:

a.i) There exist positive constants $C$ and $\gamma$ such that the weak mixing condition $\mathrm{WM}(\Lambda, C, \gamma)$ holds for every $\Lambda \subset \subset \mathbf{Z}^{\mathbf{d}}$.

a.ii) There exist positive constants $C$ and $\gamma$ such that for every $L$,

$$
\left|\mu_{\Lambda_{L}}^{+}\left(\sigma_{0}\right)-\mu_{\Lambda_{L}}^{-}\left(\sigma_{0}\right)\right| \leqq C e^{-\gamma L} .
$$

b) For all $\beta<\beta_{\text {c }}$ and all $h$ there exists positive constants $C$ and $\gamma$ such that $\mathrm{WM}(\Lambda, C, \gamma)$ holds for every $\Lambda \subset \subset \mathbf{Z}^{\mathrm{d}}$.

c) For the standard Ising model there exists a finite $\beta_{0}$ such that for all $\beta>\beta_{0}$ and all $h \neq 0$ there exists $C$ and $\gamma$ such that $\operatorname{WM}(\Lambda, C, \gamma)$ holds for every $\Lambda \subset \subset \mathbf{Z}^{\mathrm{d}}$.

Proof. a) That (a.ii) follows from (a.i) is a tautology, so we turn to the proof of the converse statement. Let $\Lambda$ be given and let $\tau, \tau^{\prime} \in \Omega_{\Lambda^{c}}$ be two boundary conditions. By the F.K.G.-Holley inequalities, (see [H] or Chapter 4 of [L]), one can construct a probability space and a family, $\left\{\sigma^{\tau} ; \tau \in \Omega_{\Lambda^{c}}\right\}$, of $\Omega_{\Lambda^{-}}$-valued random variables on this probability space so that

i) For each $\tau, \sigma^{\tau}$ has law $\mu_{\Lambda}^{\tau}$.

ii) If $\tau \leqq \tau^{\prime}$, then $\sigma^{\tau} \leqq \tau^{\tau^{\prime}}$ (both inequalities in the coordinatewise sense).

Using the relation between the total variation distance and the KantorovichRubinstein-Ornstein-Vasserstein distance (with $\varrho$ given by (1.8)), one concludes that for any $\Delta \subset \Lambda$,

$$
\begin{aligned}
\operatorname{Var}\left(\mu_{\Lambda, \Delta}^{\tau}, \mu_{\Lambda, \Delta}^{\tau^{\prime}}\right) & \leqq P\left(\sigma_{\Delta}^{\tau} \neq \sigma_{\Delta}^{\tau^{\prime}}\right) \leqq P\left(\sigma_{\Delta}^{-} \neq \sigma_{\Delta}^{+}\right) \\
& \leqq \sum_{x \in \Delta} P\left(\sigma_{x}^{-} \neq \sigma_{x}^{+}\right)=\sum_{x \in \Delta}\left|\mu_{\Lambda}^{+}\left(\sigma_{x}\right)-\mu_{\Lambda}^{-}\left(\sigma_{x}\right)\right| .
\end{aligned}
$$

Let now, for each $x \in \Delta, Q_{x}$ be the largest cube centered at $x$ and entirely contained in $A$. Then, again by F.K.G.-Holley, the r.h.s. of (3.10) can be bounded from above by:

$$
\sum_{x \in \Delta}\left|\mu_{Q_{x}}^{+}\left(\sigma_{x}\right)-\mu_{Q_{x}}^{-}\left(\sigma_{x}\right)\right| \leqq C \sum_{x \in \Delta} e^{-\gamma \operatorname{dist}\left(x, \Lambda^{c}\right)}
$$

$\operatorname{WM}(\Lambda, C, \gamma)$ follows from (3.10) and (3.11). 
b) It follows from part (i) of Theorem 2 in a paper by Higuchi, [Hi], that for all $\beta<\beta_{c}$ and all $h$ (a.ii) above is satisfied. Therefore (b) follows from (a). (Actually Higuchi, in [Hi], discusses only the standard Ising model. Nevertheless in his proof of part (i) of his Theorem 2 the only ingredients used are the GHS and Lebowitz inequalities, and the results by Aizenman, Barski and Fernandez [ABF] on the absence of intermediate phase. All these results hold in our more general situation. For the particular case $h=0$, one can also find an argument which shows that (a.ii) holds for all $\beta<\beta_{c}$ in [CCS], in the derivation of Eq. (2.7) in that paper.)

c) Again, it is sufficient to check that the condition in (a.ii) above holds. This was proved in Corollary 5.1 in [MO1] using a result by Martirosyan, [M], on the low temperature $d$-dimensional standard Ising model under arbitrary non-null external field. A stronger version of Martirosyan's result appears as Theorem 3 in [S], where the reader can find a self-contained proof in arbitrary dimension as well as a greatly simplified proof in case $d=2$.

We conclude now with a discussion of some of the nice consequences of the results presented in this paper. From Theorems 3.2 and 3.3, and the perturbative theory developed in [O, OP], one can immediately deduce, for 2D Ising models, in the one-phase region, analyticity properties of thermodynamic functions and correlation functions in terms of perturbation parameters, for a wide class of possible complex perturbations.

For simplicity only the results concerning the free energy, in the case of translation invariant complex perturbation of the Hamiltonian, are precisely stated in the following Theorem 3.4. Similar statements hold also for the expectations of local observables even in the case of non-translationally invariant complex perturbations when some spatial uniformity condition is satisfied. The following theorem is a corollary to our results above and Theorem 1.1 in [OP].

Theorem 3.4. Consider a ferromagnetic Ising model in 2D, described by the Hamiltonian given in (3.9). Let $U_{1}, \ldots, U_{l}$ be l finite range, translationally invariant real potentials (i.e. $U_{j}$ satisfy $H 1$ and $H 2$ of Sect. 1 for $j=1, \ldots, l$ ). Consider the complex partition function $Z(\Lambda, \beta, \tilde{U})$ defined as in (1.5) with $\tilde{U}=U+\sum_{j=1}^{l} \lambda_{j} U_{j}$ in place of $U$ and $\lambda_{j} \in \mathbf{C}, j=1, \ldots$, l. Let $f_{\Lambda}^{\beta}: \mathbf{C}^{l} \rightarrow \mathbf{C}$ be given by:

$$
f_{\Lambda}^{\beta}=\frac{1}{|\Lambda|} \log Z(\Lambda, \beta, \tilde{U}) .
$$

Then, for $\beta$ and $h$ as in parts (b) or (c) of Theorem 3.3 above, there exists a neighborhood $V$ of the origin in $\mathbf{C}^{\mathbf{l}}$ such that the limit

$$
\lim _{\Lambda \rightarrow \mathbf{Z}^{2}} f_{\Lambda}^{\beta}
$$

exists and is a holomorphic function of $\lambda_{j}, j=1, \ldots, l$ in $V$.

We notice that, in particular, for the standard 2D Ising model we get, from Theorem 3.4, that the free energy is a real analytic function of $h$, at $h=0$ and every inverse temperature $\beta<\beta_{c}$. To the best of our knowledge, this result, certainly expected on physical grounds, had been proven before only for $\beta \ll \beta_{c}$.

Acknowledgments. We would like to thank the Dipartimento di Matematica dell'Universita'di "Tor Vergata" and the Mathematics Department of the University of California Los Angeles for their hospitality during the early and final stage of this work. 


\section{References}

[ABF] Aizenman, M., Barski, D.J., Fernandez, R.: The phase transition in a general Ising-type models is sharp. J. Stat. Phys. 47, 343-374 (1987)

[CCS] Chayes, J., Chayes, L., Schonmann, R.H.: Exponential decay of connectivities in the two-dimensional Ising model. J. Stat. Phys. 49, 433-445 (1987)

[D] Dobrushin, R.L.: Prescribing a System of random variables by Conditional Distributions. Theory Prob. Appl. 15, 453-486 (1970)

[DS1] Dobrushin, R.L., Shlosman, S.: Constructive Criterion for the Uniqueness of Gibbs fields. Statistical Physics and Dynamical Systems, Fritz, J., Jaffe, A. and Szász, D., eds., Boston: Birkhäuser, 1985, pp. 347-310

[DS2] Dobrushin, R.L., Shlosman, S.: Completely Analytical Gibbs Fields. Statistical Physics and Dynamical Systems, Fritz, Jaffe, A. and Szász, D., eds., Boston: Birkhäuser, 1985, pp. $371-403$

[DS3] Dobrushin, R.L., Shlosman, S.: Completely Analytical Interactions. Constructive description. J. Stat. Phys. 46, 983-1014 (1987)

[DS4] Dobrushin, R.L., Shlosman, S.: Private communication. This result is attributed to Bassuev, but apparently there is no paper available with the precise results and proofs.

[G] Gross, L.: Logarithmic Sobolev Inequalities. Am. J. Math. 97, 1061-1083 (1975)

[H] Holley, R.: Remarks on the FKG Inequalities. Commun. Math. Phys. 36, 227-231 (1976)

[Hi] Higuchi, Y.: Coexistence of Infinite *-Cluster. Ising Percolation in Two Dimensions. Prob. Theory and Related Fields 97, 1-34 (1993)

[L] Liggett, T.: Interacting Particle Systems. Berlin Heidelberg New York: Springer, 1985

[LY] Lu, S.L., Yau, H.T.: Spectral Gap and Logarithmic Sobolev Inequality for Kawasaki and Glauber Dynamics. Commun. Math. Phys. 156, 399-433 (1993)

[M] Martirosyan, D.G.: Theorems on Strips in the Classical Ising Ferromagnetic Model. Sov. J. Contemp. Math. Anal. 22, 59-83 (1987)

[MO1] Martinelli, F., Olivieri, E.: Approach to Equilibrium of Glauber Dynamics in the One Phase Region I. The Attractive Case. Commun. Math. Phys. 161, 447-486 (1984)

[MO2] Martinelli, F., Olivieri, E.: Approach to Equilibrium of Glauber Dynamics in the One Phase Region II. The General Case. Commun. Math. Phys. 161, 487-514 (1984)

[MO3] Martinelli, F., Olivieri, E.: Some remarks on pathologies of renormalization group transformations for the Ising model. J. Stat. Phys. 72, 1169-1178 (1993)

[O] Olivieri, E.: On a cluster Expansion for Lattice Spin Systems a Finite Size Condition for the Convergence. J. Stat. Phys. 50, 1179-1200 (1988)

[OP] Olivieri, E., Picco, P.: Cluster Expansion for D-Dimensional lattice Systems and Finite Volume Factorization Properties. J. Stat. Phys. 59, 221-256 (1990)

[S] Schonmann, R.: Slow droplet-driven relaxation of stochastic Ising models in the vicinity of the phase coexistence region. Commun. Math. Phys. 161, 1-49 (1994)

[Sh] Shlosman, S.B.: Uniqueness and Half-Space Nonuniqueness of Gibbs States in Czech Models. Theor. Math. Phys. 66, 284-293 (1986)

[SZ] Stroock, D.W., Zegarlinski, B.: The Logarithmic Sobolev Inequality for Discrete Spin Systems on a Lattice. Commun. Math. Phys. 149, 175-194 (1992) 
